\title{
BRECHA SALARIAL POR GÉNERO EN MÉXICO: DESDE UN ENFOQUE REGIONAL, SEGÚN SU EXPOSICIÓN A LA APERTURA COMERCIAL 2005-2015
}

Gender pay gap in Mexico: From a regional perspective, according to its exposure to the 2005-2015 trade opening

Reyna Elizabeth Rodriguez Pérez ${ }^{1}$

Fecha de recepción: 09 de agosto de 2016 Fecha de aceptación: 21 septiembre de 2016

1- Nombre: Reyna Elizabeth Rodriguez Pérez. Nacionalidad: Mexicana. Grado: Doctorado en ciencias. Especialización: Desigualdad salarial. Adscripción: Universidad Autónoma de Coahuila. Correo electrónico: reynarodriguez@uadec.edu.mx 


\section{Resumen}

El objetivo de esta investigación es analizar la brecha salarial por género a lo largo de la distribución salarial en las regiones de México. Para ello se aplica, la metodología de Melly (2005) con la Encuesta de Ocupación y Empleo (ENOE) 2005-2015. Los resultados revelan que, en México, y sus regiones la desigualdad salarial por género, ha disminuido, aunque esta se encuentra en contra de las mujeres. La mayor parte de las diferencias no se explican por las características productivas, acentuándose en la parte alta de la distribución salarial, en las regiones más expuestas a la apertura comercial.

Palabras clave: Economía laboral, diferencias salariales, regiones.

\section{Abstract}

The objective of this research is to analyze the gender wage gap throughout the wage distribution in regions of Mexico. This applies to the methodology Melly (2005) Survey of Occupation and Employment (ENOE) 2005-2015. The results show that in Mexico, and its regions gender wage inequality has declined, although this is against women. Most of the differences can not be explained by productive characteristics, accentuating at the top of the wage distribution in the most exposed to trade liberalization regions.

Keywords: Labor economics, wage differentials, regions. 


\section{Introducción}

S egún la OCDE (2008: 310) México es una economía emergente con una elevada desigualdad. En las últimas décadas ha experimentado grandes transformaciones, una de ellas es la apertura comercial, desde la firma del Acuerdo General sobre Aranceles Aduaneros y Comercio (GATT) hasta El Tratado de Libre Comercio de América del Norte (NAFTA), sin embargo, existen grandes diferencias regionales y locales en cuanto al uso de los recursos productivos, la actividad económica y al nivel de vida que se presentan, desde tiempos previos a la apertura comercial.

Hoy en día, a casi 30 años de que México se incorporase a la Organización Mundial del Comercio (OMC) y a más de 20 años de la firma del Tratado de Libre Comercio de América del Norte (TLCAN), se ha generado una relocalización de las actividades económicas del país, provocando que ya no sea únicamente la región del centro el motor económico más importante. Esta relocalización ha traído consigo que no todas las regiones sean capaces de atraer inversión, que coadyuve al crecimiento económico y empleo, por lo tanto, los trabajadores de cada una de las regiones sufren de manera distinta, grandes desigualdades económicas, al impactar gravemente el salario de la mano de obra, principalmente el de las mujeres.

El aumento de las mujeres en el mercado laboral en México se relaciona con la globalización y principales efectos. La participación femenina en el mercado laboral pasó de 17.6 por ciento en 1970 a un 41.8 por ciento en la actualidad, según el Censo de Población de 2010 (INEGI, 2010), el 51.2 por ciento de la población total son mujeres, de las cuales el 42.5 por ciento forman parte de la población económicamente activa, y de este universo el 94.8 por ciento se encuentran ocupadas, con un nivel de ingreso promedio equivalente al 80 por ciento respecto al hombre (Rodríguez y Castro (2014a: 655-686)). Asimismo, se destaca que solo el 1.41por ciento de las mujeres tienen puestos de toma de decisión como gerentes o funcionarias públicas y el 15.0 por ciento de ellas se desempeña como profesionista o técnico especializado, el 26.9 por ciento se dedica a actividades comerciales, como vendedoras (ENOE 2015).

Aunque existe una mayor incorporación de la mujer al mercado de trabajo, ellas siguen sufriendo una elevada desigualdad no explicada por sus atributos productivos. Se han hecho innumerables investigaciones a nivel internacional sobre el tema de la desigualdad salarial por género, aunque se han indagado poco la perspectiva regional, de las investigaciones encontradas se puede mencionar el de Enamorado et al. (2009) para Centroamérica; Galvis (2011) para Colombia; Liu et al. (2000) para Shanghái y Jinan, China y Hirsch (2013) para Alemania; Del Río et al. (2008) referente a comunidades de España, así como el de Ñopo et al. (2011), en la mayoría de los trabajos se destaca el hecho de que las mujeres siguen obte-niendo menores salarios en relación a los hombres, aunque tengan los mismos o más atributos productivos como educación y experiencia.

En México se han elaborado investigaciones con enfoque regional a cargo de Mendoza y García (2009); Rodríguez y Castro (2014a); así como Castro et al. (2015), donde las brechas salariales por género se encuentran a favor de los hombres. Todos los estudios que han analizado el tema, han destacado que los hombres reciben una remuneración mayor que las mujeres, a pesar de que ellas han aumento sus años educativos y su participación en el mercado de trabajo. Por lo tanto ¿cómo se puede explicar que esta diferencia exista? 
Para poder contestar esta pregunta varios autores han analizado las brechas salariales por género y su descomposición, la mayor parte de los trabajos utilizan el método tradicional Oaxaca-Blinder (1973), no obstante en los últimos tiempos se ha realizado la descomposición de la brecha de salarios con metodologías no paramétricas DiNardo et al. (1996) y paramétricas Machado y Mata (2005) y Melly (2005) a lo largo de toda la distribución de salarios con corrección de sesgo de selección (Heckman 1979). De igual manera se ha indagado sobre los factores que explican los cambios en la parte explicada por los atributos de capital humano en términos de características y los precios Chinhui et al. (1991, 1993). Aunque se ha abordado poco sobre el efecto de la apertura en las brechas de salario en las regiones del país. La motivación de este estudio se basa en conocer si la explosión a la apertura comercial en las regiones de México ha impactado las brechas de salario por género de 2005 a 2015.

Resulta también interesante explorar si ¿existen cambios a lo largo de toda la distribución de salarios? ¿En qué segmento de la distribución existen más diferencias? ¿y si estas diferencias varían por regiones?

Por tanto, el objetivo de esta investigación es analizar las diferencias salariales y su descomposición a lo largo de la brecha de salarios en las regiones de México, ante su exposición a la apertura comercial en el periodo 2005-2015. Aplicando la metodología de Melly (2005) basada en una regresión cuantílica y su descomposición y la regionalización de Aguilera y Castro (2016), con los datos de la Encuesta de Ocupación y Empleo (ENOE) 2005-2015.

La hipótesis que se desea contrastar se basa en que las mujeres ubicadas en las regiones más expuestas a la apertura comercial, experimentan una reducción de la brecha a su favor, explicada mayormente por las características productivas, en los cuantiles más altos de la distribución salarial.

Los principales hallazgos de esta investigación, indican que las brechas de salarios se han reducido de 2005 al 2015, a favor de los hombres, aunque se ha encontrado gran heterogeneidad a nivel regional. En 2005 , las regiones de alta y media exposición reflejan el fenómeno de "techo de cristal" y se mantiene para la última región en 2015, debido al aumento en el valor de los coeficientes. Asimismo, se encontró que en 2005 y 2015 la región de baja exposición a la apertura comercial, muestra reducción de la desigualdad al avanzar en los cuantiles de salario, debido a una reducción del valor de los coeficientes. Por lo anterior se rechaza la hipótesis propuesta, ya que el mercado laboral, resultó más favorable para las mujeres que se encuentra en las regiones de baja exposición a la apertura comercial.

Este trabajo presenta el siguiente orden: el segundo apartado describe algunas teorías sobre apertura comercial, desigualdad salarial y evidencia empírica. En la tercera parte se describen los datos y la metodología para este estudio. El cuarto presenta los resultados del análisis empírico sobre la descomposición de la desigualdad salarial. En el último apartado se detallan las conclusiones y la bibliografía utilizada.

\section{Revisión de literatura y conceptos}

\subsection{Apertura comercial y desigualdad}

La teoría del equilibrio general del comercio aportado por Eli Heckscher y Bertil Ohlin abrió una nueva línea de investigación "enfocada en las diferencias de la intensidad relativa de factores a través de la industria y las diferencias en la abundancia relativa de factores a través de los países”(McCulloch, 2006:3). 
El teorema realizado por Wolfgang Stolper y Paul Samuelson en 1941, el cual es uno de los resultados centrales de la teoría de Heckscher-Ohlin (Neary, 2004), parte del supuesto, en donde dos países tienen dotación de factores diferentes, algunos con abundancia en capital y otros con abundancia de trabajo, gracias a la especialización internacional, los primeros producirán bienes intensivos en capital, mientras que los segundos se especializarán en bienes intensivos en trabajo. Esto tendrá como resultado un efecto sobre la distribución de la renta, resultando "ganador" el factor más abundante en el país, siendo el comercio internacional un mecanismo "sustituto" de movilidad de factores y en el largo plazo la consecuencia será la igualación de precio de los factores entre países. Por tanto, cada país exportará los productos que están hechos con su factor abundante de producción e importará aquellos donde es escaso.

El proceso de apertura comercial tiene efectos remarcables sobre las economías de los países a nivel de reestructuración regional, donde aquellas con mejor infraestructura física, capital humano e instituciones adecuadas, presentarán un mayor crecimiento en comparación con regiones que carecen de tales características. (Aguilera y Castro 2016). Por otro lado, las regiones con mayores rezagos económicos, deben alcanzar un nivel mínimo de desarrollo en educación, salud e infraestructura para atraer elevados flujos de Inversión Extranjera Directa (IED) y de este modo evitar la excesiva concentración económica, centralidad de decisiones políticas y desigual distribución de beneficios.

La desigualdad, entendida como la brecha de nivel de vida entre personas, es un tema que en décadas recientes ha vuelto al centro del análisis económico. Gasparini (2006) menciona que el mundo ha visto una importante reducción de la pobreza en términos relativos, sin embargo, en las últimas décadas el crecimiento de la desigualdad es un fenómeno que se presenta en común en países desarrollados y en desarrollo (Castro y Huesca, 2007).

Existen diferentes hipótesis sobre las causas de la brecha de salarios, las cuales se sustentan en los cambios de la demanda, la oferta, transformaciones tecnológicas, cambios en las instituciones o bien, gracias a la influencia del comercio internacional. De igual manera se han hecho análisis sobre la desigualdad salarial por regiones, tanto en el ámbito internacional como en el nacional, dicho de otro modo, que la desigualdad salarial no es la misma cuando se comparan distintos países, o distintas zonas geográficas dentro de una mismo continente o país (Castro y Huesca, 2007).

En esta investigación se analizarán la diferenciación salarial y su descomposición por género. En Rodríguez y Castro (2014a) se menciona que por razones históricas de carácter cultural, los estudios de género se enfocan principalmente desde la perspectiva femenina, sin embargo, debe aclararse que el concepto de desigualdad por género, es sólo una dimensión de un problema más complejo, que se manifiesta en muchas otras formas de discriminación y segregación que frecuentemente se intersectan en la vida cotidiana, para convertirse en limitantes en la búsqueda de formas de convivencia más justas y satisfactorias de interacción para la sociedad en su conjunto.

Las conductas discriminatorias practicadas por distintos agentes, como consumidores y productores, se plasma en la teoría del gusto por la discriminación desarrollada por Becker (1967), según este autor, en base a sus preferencias algunos individuos actúan como si tuvieran una disponibilidad a pagar para evitar realizar transacciones con determinados colectivos, dado que ello le implica un costo subjetivo o psicológico.

Por lo anterior, se consideró importante contar con una estimación de la magnitud de la desigualdad salarial y su descomposición entre hombres y mujeres ocupados(as) en regiones de México según su 
exposición a la apertura comercial, si es que tiene lugar, con el fin de confirmar si el grupo femenino obtiene un salario promedio menor que la población masculina por razones diferentes a sus características productivas. Ante esta inquietud, presentamos a continuación las generalidades de los datos, su tratamiento y los hallazgos observados de diferentes estudios que abordan el problema a nivel internacional y para México.

\subsection{Evidencia empírica}

El tema ha sido analizado a nivel internacional desde la perspectiva regional por Blau y Kahn (1996) para algunos países de Europa (Austria, Alemania, Hungría, Suiza, Reino Unido, Noruega, Suecia, Australia); Semykina y Linz (2005) para Rusia, Alemania y Kazakhstan; Enamorado et al. (2009) para Centroamérica (Costa Rica, Honduras, Nicaragua, El Salvador); Galvis (2001) para Colombia; Liu et al. (2000) para Shanghái y Jinan, China; Mysicova (2012) para Republica Checa, Hungría, Polonia y Eslovaquia; Hirsch (2013) para Alemania; Del Río et al. (2008) referente a comunidades de España, así como el de Nopo et al. (2011), para 64 países, Wolszczak-Derlacz (2013) para 18 países miembros de la OCDE, los resultados varían por regiones, aunque en la mayoría de ellos se destaca el hecho de que las mujeres siguen obteniendo menores salarios en relación a los hombres, aunque tengan los mismos o más atributos de capital humano.

El análisis de la desigualdad salarial por género ante los efectos de la apertura comercial, en regiones y sectores de México, se ha realizado por Artecona y Cunningham (2002) y Domínguez y Brown (2010). Las primeras, analizando la Encuesta Nacional de Empleo Urbano (ENEU), la Encuesta Nacional de Empleo, Salarios, Tecnología y Capacitación (ENESTyC) y la Encuesta Industrial Mensual (EIM), para la primera fase de liberalización comercial (1987-1993), concluyen que con la liberalización comercial se asocia una alta brecha de género en el sector manufacturero de México, sin embargo, recalcan que cuando se quita el efecto de la prima por experiencia a los hombres, la evidencia sugiere que la liberalización comercial favorece la disminución de la discriminación salarial. Las segundas, tomando en cuenta únicamente datos de la ENESTyC y un periódo más reciente (2001-2005), infieren que existe un impacto negativo de la orientación exportadora en los salarios de los hombres y las mujeres, además en el ratio de salario de género, donde la mujer es mayormente penalizada en términos absolutos y relativos.

Martínez y Acevedo (2004) quienes realizaron un estudio basado en la Encuesta Nacional de Ingreso Gasto de Hogares (ENIGH) 2000, realizaron un análisis de descomposición salarial con el método de Oaxaca (1973)-Blinder (1973), su estudio reveló la existencia desigualdad a favor de los hombres, en las áreas urbanas como rurales del país, con más del 85.0 por ciento de la desigualdad es asociado con la parte no explicada por los atributos productivos.

Tello et al. (2012) con los datos de la ENOE y ENEU 1987-2008, analizan la relación entre la inequidad salarial, la estructura de empleo y los retornos a la educación, aplicando la metodología de Melly (2005), al realizar la descomposición de la desigualdad salarial por cuantiles, así como el análisis de las covarianzas individuales para áreas urbanas en México. En sus resultados encuentran que las diferencias en las características son mucho más importantes en la parte inferior (percentil 10) que en la parte superior (percentil 90) de la distribución salarial. De hecho, algunos de los efectos significativos de la estructura salarial emergen en el percentil 90. 
Popli (2013) realiza un análisis comparativo del diferencial salarial por género entre 1996 y 2006, con los datos de la ENIGH, distinguiendo entre el mercado laboral formal e informal para México. Para ello utiliza la metodología paramétrica y no paramétrica con función de la expectativa condicional loglineal. El autor encuentra que la brecha salarial por género es más alta en el mercado de mano de obra formal, aunque de manera general las diferencias salariales han disminuido en el tiempo (1996-2006) en ambos mercados, de igual manera se encuentra evidencia de techo de cristal en el formal, con un aumento en la parte no explicada principalmente en la cola superior de la distribución de ingresos.

Rodríguez y Castro (2014a) obtienen la brecha de salarios por género y su descomposición para México y sus regiones en el periodo 2000-2004, con los datos de la ENEU, al aplicar la metodología Oaxaca (1973) y Blinder (1973), sus resultados indican que existen diferencias salariales en contra de las mujeres en más del 85 por ciento, que no se deben a las características observables de capital humano, este comportamiento es heterogéneo a nivel regional con oscilaciones entre 15 y 25 por ciento de desigualdad. La parte no explicada por las características tiende a converger en 20 por ciento, con excepción de la capital del país que se ubica en 13 por ciento.

En otro estudio de Rodríguez y Castro (2014b) obtienen que para el caso de la manufactura de las ciudades de Saltillo y Hermosillo con los datos de la ENOE 2005-2011, al utilizar la metodología de Oaxaca (1973)-Blinder (1973), encuentran que en Saltillo y Hermosillo las diferencias son de 26.0 y 27.0 por ciento en promedio para el periodo, a favor de los hombres, y la posterior descomposición del ingreso confirmaron que las tres cuartas partes de la diferencia de ingreso en estas áreas metropolitanas, están asociadas con la parte no explicada por las características.

Por su parte, Arceo y Campos (2014) analizan la brecha por género para el periodo 1990-2010, utilizando los Censos de Población y encuentran que en promedio existe una disminución de la desigualdad en el periodo. Al utilizar la metodología semiparametrica propuesta por DiNardo et al. (1996) para descomponer la brecha y corregir por sesgo de selección de las mujeres en el mercado laboral, encuentran que la mayor parte de la brecha salarial se debe al efecto de los precios y no de características, sin embargo, al corregir por sesgo la brecha salarial hubiera sido mayor para las mujeres de baja educación y en cuantiles bajos.

Castro et al. (2015) analizan la discriminación salarial por género, en la industria manufacturera de la frontera norte de México durante en el periodo 2005-2011, con información de ENOE. Utilizan la técnica Oaxaca (1973)-Blinder (1973) y corrigen por sesgo de selección, sus resultados indican que tanto a nivel frontera norte como nacional, en la industria, las mujeres tienen percepciones salariales, aproximadamente 13 por ciento menos, con respecto a los hombres por motivos diferentes a sus atributos productivos.

En resumen, la revisión de evidencia empírica esbozada en este apartado, cubre desde el 2000 hasta el 2015, considerando diferentes fuentes de información, así como distintos enfoques metodológicos, destacando el método de Oaxaca (1973)-Blinder (1973) como el más frecuente, no obstante en los años recientes se han explorado enfoques alternativos para descomponer la brecha salarial, buscando identificar algunos fenómenos en el comportamiento de las diferencias de ingreso por género a lo largo de la distribución, como pueden ser la presencia "techo de cristal" y/o "piso pegajoso".2 
De igual manera, gran parte de los autores concluyen que durante el periodo de apertura comercial y posterior, se ha presentado en México un aumento en la desigualdad en contra de las mujeres, sin embargo, aún no se ha llegado a un consenso sobre cuál ha sido el factor determinante, empero, son pocos los trabajos que han analizado las diferencias por género, desde la perspectiva regional según su exposición a la apertura comercial.

\section{Datos y metodología 2.1 Datos}

El estudio se basa en el análisis de los datos individuales de la ENOE realizada de manera trimestral por el Instituto Nacional de Estadística y Geografía (INEGI). En el presente trabajo se considera el tercer trimestre de los años 2005 y 2015, lo que evita los efectos de factores estacionales y con lo cual se pretende cubrir el periodo de información disponible más reciente de la encuesta y a la vez, extender el análisis de estudios previos similares.

La población de estudio son mujeres y hombres subordinados y remunerados entre 16 y 65 años de edad. Se utilizan los años de educación, así como la experiencia potencial de los individuos, así como, el salario mensual por hora deflactado con el Índice de Precios al Consumidor (IPC) 2015.

Existen diferentes trabajos que clasifican a México en regiones con énfasis en el grado de exposición a la apertura comercial, como Hanson (2005) y Gutiérrez (2008), sin embargo, en esta investigación se consideró la regionalización propuesta por Aguilera y Castro (2016), por considerarse la más completa y acorde con el análisis que se desea realizar, ya que clasifican a los estados que tienen alta, media o baja exposición a la apertura comercial, mediante su especialización productiva y su capacidad exportadora e importadora a partir de estimar coeficientes de localización y de exportación, calculados con datos del PIB a precios de 2003 para el periodo 2005-2011. Los autores seleccionaron como estados de alta exposición aquellos que según su rango se mantuvieron mayormente en el tercio superior en las medidas de participación de las industrias manufactureras en el PIB estatal, flujos de inversión extranjera directa, valor agregado censal bruto per cápita del sector manufacturero, porcentaje del empleo en las manufacturas sobre el total del empleo estatal y distancia a los Estados Unidos. Por el contrario, se consideraron como de baja exposición las entidades que permanecieron en el tercio inferior, y, por consiguiente, las restantes como de media exposición.

Las regiones a considerar son las siguientes: Región de alta exposición: Aguascalientes, Baja California, Chihuahua, Coahuila, Estado de México, Guanajuato, Querétaro, Nuevo León, Puebla, San Luis Potosí, Sonora y Tamaulipas; Región de media exposición: Baja California Sur, Distrito Federal, Durango, Hidalgo, Jalisco, Michoacán, Morelos, Tlaxcala, Veracruz y Zacatecas y Región de baja exposición: Campeche, Chiapas, Colima, Guerrero, Nayarit, Oaxaca, Quintana Roo, Sinaloa, Tabasco y Yucatán.

superior de la distribución, indicando la existencia de un tope o techo de ingresos para las mujeres y por "piso pegajoso" se entiende la presencia de una brecha salarial significativamente alta en la parte inferior de la distribución. 


\subsection{Descomposición de las diferencias en la distribución salarial por cuantiles}

Para cumplir con el objetivo de esta investigación, se estiman ecuaciones de salario usando una técnica de regresión cuantílica, para diferentes tramos de la distribución salarial, con el objetivo de descomponer las diferencias salariales entre mujeres y hombres, en sus componentes explicados y no explicados por las características de capital humano.

Koenker y Bassett (1978) y Koenker y Hallock (2001), proponen la técnica de regresión cuantilica, mostrando los determinantes del salario en diferentes tramos de la distribución. Dueñas et al. (2015) menciona que el propósito de la técnica de regresión cuantilica es aproximar la media condicionada y otras variables de respuesta cuantílica como medidas de dispersión, como es el caso de la varianza, desviación estándar, entre otras. El mismo autor señala que este tipo de estimaciones son apropiadas cuando el comportamiento que se intenta estudiar, no presenta las mismas pautas en todas y cada una de las partes de la distribución, o si la misma incluye casos de interés atípicos.

Por su parte, Melly (2005) toma la técnica de regresión de Mincer (1974) al realizar el análisis por cuantiles, basado en el aporte de Koenker y Bassett (1978) y Koenker y Hallock (2001), desde el enfoque contrafactual de Oxaca-Blinder (1973).

Melly (2005), analiza los efectos de las covarianzas sobre la desigualdad salarial y su descomposición en cada uno de los cuantiles, donde muestra si tales diferencias se deben a los efectos en las características o por los coeficientes. Se calculan las regresiones por cuantiles por separado para cada año de estudio para $\hat{q} \operatorname{con} \theta=0.10,0.20,0.30,0.40,0.50,0.60,0.70,0.80,0.90$.

$$
\hat{q}_{1}(\theta)-\hat{q}_{0}(\theta)=[\hat{q}(\theta)-\hat{q}(\theta)]+[\hat{q} c(\theta)-\hat{q}(\theta)]
$$

Donde el primer término es la parte de la disparidad del logaritmo de salarios por hora, debido a las diferencias de las características (educación continua y experiencia potencial) y entre hombres y mujeres.

$$
\hat{q}_{1}(\theta)=\inf \left\{q: n_{t}^{-1} \sum_{i: T_{i}=t} \hat{F}_{Y(t)}\left(q \mid X_{i}\right) \geq \theta\right\}
$$

El segundo término es la parte de la desigualdad asociada con la diferencia en las remuneraciones a las características, es decir corresponde a la parte de la desigualdad del logaritmo de salarios, debido a las diferencias (cuantiles) de las características entre hombres y mujeres. La cual se explica por la función cuantílica no condicionada basada en un tratamiento binario de $T$ (mujeres 0 y hombres 1 ) y $C$ reflejaría la función contrafactual, $Y$ refleja la variable explicativa del logaritmo de salarios por hora, $X$ son las variables explicativas para el individuo $i$, en este caso educación y experiencia potencial y $F_{Y}(q)$ es la función acumulada.

$$
\hat{q} c(\theta)=\inf \left\{q: n_{t}^{-1} \sum_{i: T_{i}} F_{Y(0)}\left(q \mid X_{i}\right) \geq \theta\right\}
$$


La descomposición del diferencial salarial con la técnica de Oaxaca (1973) y Blinder (1973), supone la ausencia de discriminación y se parte del supuesto de que los efectos generados por las dotaciones de capital humano deberían ser idénticos para ambos grupos; de forma que las diferencias en las remuneraciones solo reflejen disparidades en las dotaciones de capital humano, y por tanto en la productividad laboral.

Para explicar los cambios en la estructura de los salarios, la teoría económica estándar se centra en la dinámica salarial promedio en lugar de los cambios en toda la distribución salarial, ignorando así las diferencias en la parte inferior o la parte superior de la distribución salarial. Además, las estadísticas salariales promedio pueden ocultar funciones importantes en la estructura salarial. La contribución de esta investigación es obtener las diferencias salariales a lo largo de toda la distribución, por cuantiles a nivel de regiones según su exposición a la apertura comercial en México en la década reciente.

\section{Resultados}

\subsection{Estadística descriptiva}

En esta sección se presenta la estructura ocupacional y salarial del mercado laboral mexicano y sus diferentes regiones, según su exposición a la apertura comercial en 2005-2015. El cuadro 1, revela que, en términos salariales, la remuneración promedio por hora ha caído en la década en todo el país y en cada una de las regiones. A nivel espacial, se observa que en la región de alta exposición a la apertura se cuenta con un mayor salario por hora, aunque en esta zona es donde se experimenta una mayor caída (43.46 a 35.86), respecto al resto del país. De igual manera, se encontró que la proporción de jefe de hogar, oscila alrededor de 45.0 puntos porcentuales y se mantiene relativamente sin cambios, al igual del estatus civil, donde poco más del 60.0 por ciento de los trabajadores es casado. Por otra parte, el promedio de años de educación se mantiene constante, aunque, son los trabajadores ubicados en la región de baja exposición, donde se observan mayores años educativos con 11.05 en promedio en 2015. La variable experiencia muestra un ligero aumento de 2005-2015 en todas las regiones de estudio, al pasar a más de 20 a 21 años promedio.

De igual manera, existe un mayor porcentaje de trabajadores calificados, en la región de media exposición en cada uno de los años analizados, con más del 40.0 por ciento. En términos del sexo, la mayor proporción de trabajadores en cada una de las regiones corresponde al masculino con más del 60.0 por ciento de participación, sin embargo, cuando se analiza esta proporción a través del tiempo se observa que hay una disminución de este indicador, por lo que deduce que poco a poco las mujeres han ganado participación en el mercado de trabajo, principalmente en la región de alta exposición con un aumento de 4 puntos porcentuales.

Asimismo, existe una mayor participación en el grupo de 26 a 35 años en 2005, no obstante, esta proporción cambia en 2015, ya que se incrementa la participación del grupo de 36-45 años, lo cual se debe a que el grupo de trabajadores más jóvenes de 16-25 ha perdido participación en el último año de estudio, una de las posibles razones es que muchos individuos están postergando su entrada al mercado de trabajo, ya que se encuentran estudiando alguna carrera universitaria. 
Se encontró, que existe una mayor proporción de trabajares en la región de media exposición con más del 50.0 por ciento, esta proporción se mantiene relativamente sin cambios en 2015. Los sectores económicos con mayor peso son la industria manufacturera, el comercio y los servicios diversos. A nivel regional es importante resaltar que, en la región de alta exposición, es donde se concentra la actividad manufacturera del país con más del 25.0 por ciento en el periodo de estudio.

Cuadro1. Variables promedio para México y regiones según su exposición a la apertura comercial 2005-2015

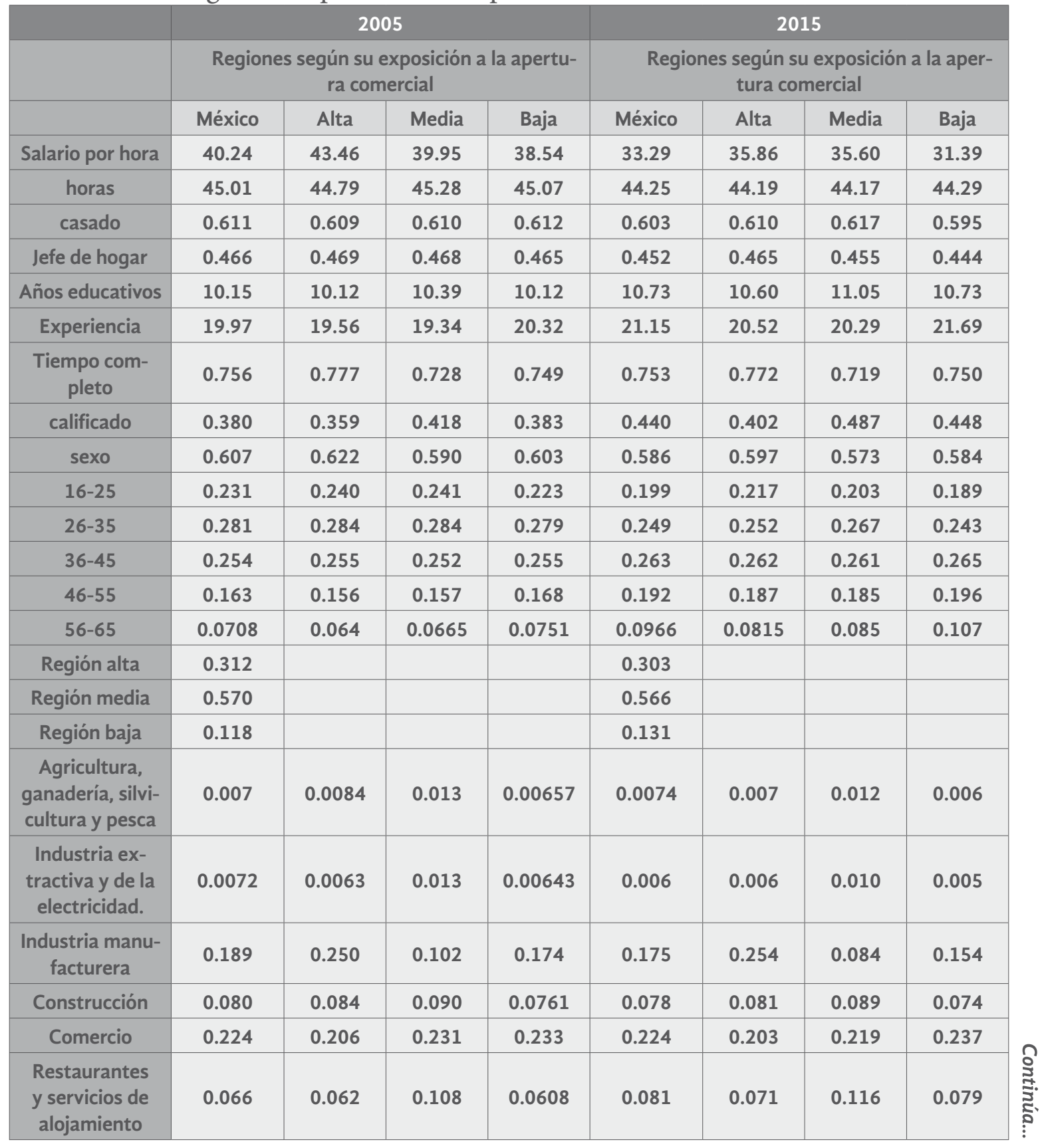




\begin{tabular}{|c|c|c|c|c|c|c|c|c|}
\hline & \multicolumn{4}{|c|}{2005} & \multicolumn{4}{|c|}{2015} \\
\hline & \multicolumn{4}{|c|}{$\begin{array}{l}\text { Regiones según su exposición a la apertu- } \\
\text { ra comercial }\end{array}$} & \multicolumn{4}{|c|}{$\begin{array}{c}\text { Regiones según su exposición a la aper- } \\
\text { tura comercial }\end{array}$} \\
\hline & México & Alta & Media & Baja & México & Alta & Media & Baja \\
\hline $\begin{array}{l}\text { Transportes, co- } \\
\text { municaciones, } \\
\text { correo y alma- } \\
\text { cenamiento }\end{array}$ & 0.073 & 0.061 & 0.065 & 0.0808 & 0.065 & 0.055 & 0.060 & 0.072 \\
\hline $\begin{array}{c}\text { Servicios } \\
\text { profesionales, } \\
\text { financieros }\end{array}$ & 0.084 & 0.080 & 0.072 & 0.0881 & 0.100 & 0.091 & 0.090 & 0.107 \\
\hline $\begin{array}{l}\text { Servicios so- } \\
\text { ciales }\end{array}$ & 0.0857 & 0.0815 & 0.0920 & 0.0867 & 0.0851 & 0.0836 & 0.100 & 0.082 \\
\hline $\begin{array}{l}\text { Servicios diver- } \\
\text { sos }\end{array}$ & 0.121 & 0.112 & 0.126 & 0.124 & 0.119 & 0.108 & 0.133 & 0.122 \\
\hline Gobierno & 0.0612 & 0.0470 & 0.0860 & 0.0639 & 0.056 & 0.039 & 0.082 & 0.060 \\
\hline Observaciones & $15,930,807$ & $4,970,825$ & $1,874,442$ & $9,085,540$ & $16,951,136$ & $5,138,287$ & $2,214,295$ & $9,598,554$ \\
\hline
\end{tabular}

Fuente: Elaboración propia en base a los datos de la ENOE, años respectivos.

\subsection{Regresiones por cuantiles}

En este apartado se realiza el análisis por regresiones cuantílicas para México y en regiones según su exposición a la apertura comercial, en el periodo 2005-2015. Con el objetivo de analizar el comportamiento de los coeficientes de educación y experiencia para hombres y mujeres a través del tiempo y en regiones. En la gráfica 1, se muestra que en México y en cada una de las regiones, los coeficientes de educación son mayores en el grupo de mujeres, en todos los cuantiles de 2005 a 2015, asimismo, se encontró que los coeficientes se reducen significativamente para ambos gr upos de sexos en 2015, au nque parece haber menos diferencias en los cuantiles bajos y altos de la distribución salarial e incluso en algunos tramos de la distribución, el premio de las mujeres en 2015, iguala al de los hombres en 2005.

El análisis por regiones, revela que en la zona de alta exposición el premio a la educación de las mujeres en 2015, es similar al grupo de hombres de 2005, en los cuantiles IV al VIII. Por su parte, en las regiones de media y baja exposición se observa que los coeficientes de hombres de 2005, tienden a igualarse con ese mismo grupo en 2015, en diferentes tramos de la distribución, como es el caso de la región de media exposición en los cuantiles II al VI y en la región de baja exposición en los primeros I y II, así como en el último cuantil.

Con lo anterior se puede deducir que el premio a la educación ha bajado para mujeres y hombres en todas las regiones de 2005 a 2015, afectado principalmente a los trabajadores ubicados en la parte alta y baja de la distribución de salarios. 
Gráfica 1. Coeficientes de escolaridad en cuantiles por sexo en México y sus regiones según su exposición a la apertura comercial. 2005-2015

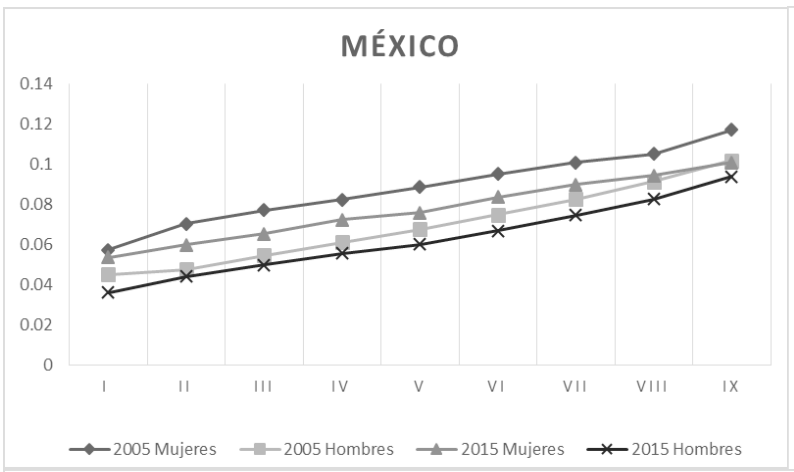

REGIÓN MEDIA EXPOSICIÓN

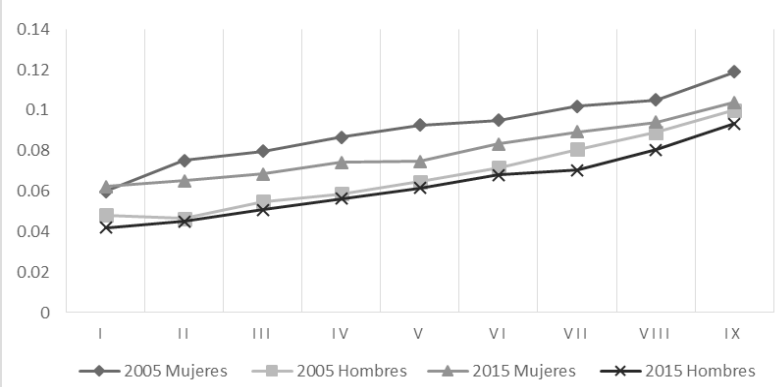

REGIÓN ALTA EXPOSICIÓN

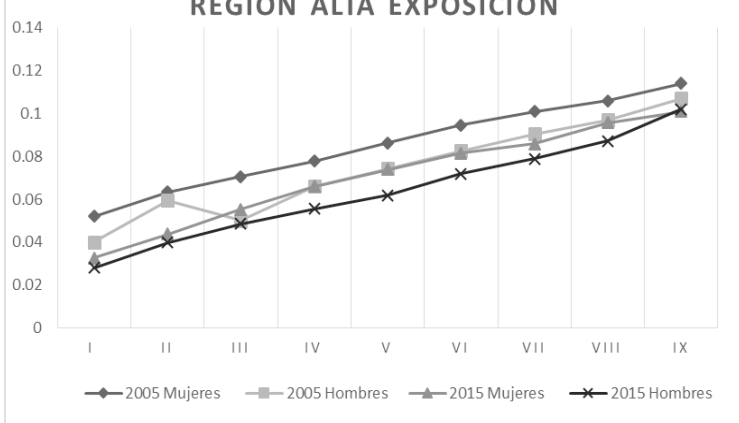

REGION BAJA EXPOSICIÓN

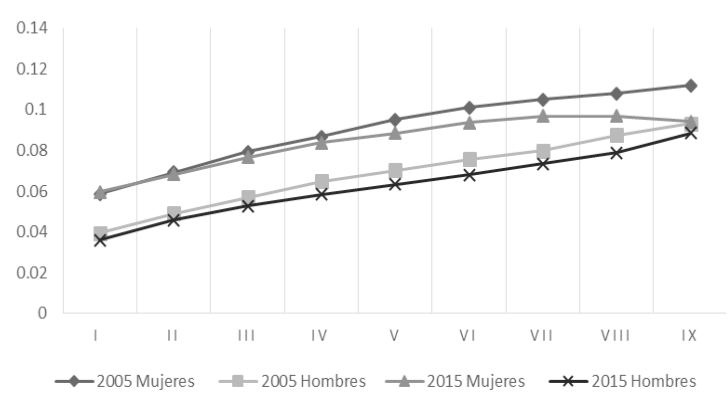

Fuente: Elaboración propia en base a los datos de la ENOE, años respectivos.

En la gráfica 2, se muestra que a nivel nacional los coeficientes de la experiencia son mayores en el grupo de hombres, principalmente en 2005, ya que, en 2015, esta variable tiende a homologarse entre sexos, siendo mayor para ellas en los cuantiles VI y VII de la regresión de salarios.

Todas las regiones muestran un comportamiento similar al nacional, no obstante, cuando se indaga sobre el comportamiento de los coeficientes por cuantiles, se observa que en la región de alta exposición el premio a la experiencia tiende a igualarse en los primeros cuantiles y en los últimos en 2005 y en 2015. Para la región de media exposición, los precios de la experiencia en 2015 favorecen a las mujeres respecto a los hombres, no obstante, se aprecia que la experiencia, también tiende a converger entre grupos en los primeros y últimos cuantiles de salario en 2005 y 2015. Por su parte, en la región de baja exposición a la apertura comercial los coeficientes han caído de 2005 a 2015, para mujeres y hombres. En 2015 el premio a la experiencia es mayor para las mujeres, aunque tiende a homologarse en los últimos cuantíles del 2015.

Con lo anterior se puede deducir que el precio a la experiencia ha bajado para mujeres y hombres en todas las regiones, aunque se observa que los coeficientes tienden a ser mayores para las mujeres, así como a homologarse en 2015 , afectado principalmente a los trabajadores ubicados en la parte alta y baja de la distribución de salarios. 
Gráfica 2. Coeficientes de experiencia en cuantíles por sexo en México y sus regiones según su exposición a la apertura comercial. 2005-2015

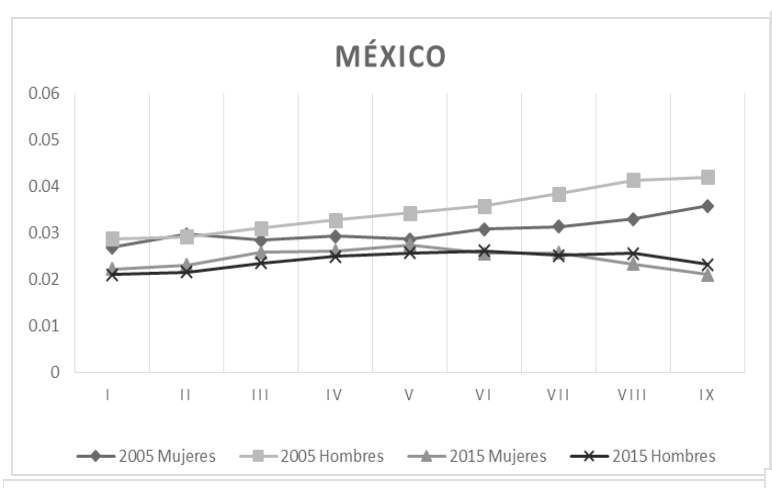

REGIÓN MEDIA EXPOSICIÓN

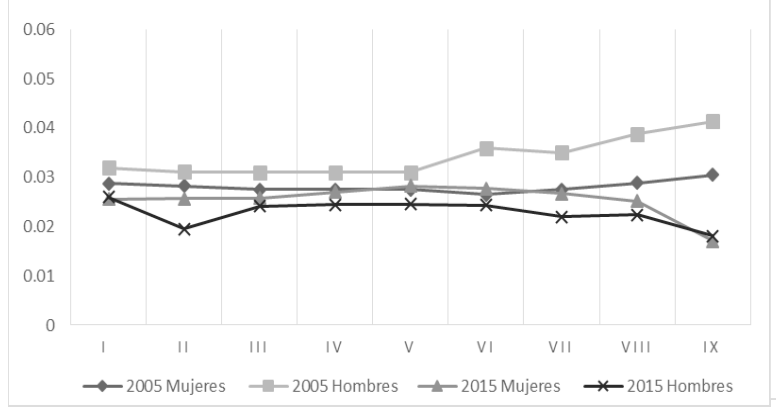

Fuente: Elaboración propia en base a los datos de la ENOE, años respectivos.

En resumen, se encontró que el premio a la educación es mayor para las mujeres, aunque no sucede lo mismo cuando se analiza la experiencia, ya que esta se muestra a favor de los hombres, sin embargo, en esta última variable la región de media y alta exposición muestran mayores rendimientos para las mujeres. Un hallazgo importante es que en México el precio a la educación y experiencia es mayor en 2005 respecto al 2015, por lo que se deduce que el precio de las características ha caído a través del tiempo principalmente en 2015. Así mismo, los resultados revelan que en los primeros y en los últimos cuantiles de salarios, los precios en educación y experiencia en términos de coeficientes tienden a converger entre sexos, en ambos años de estudio.

Por los resultados mostrados, no se puede conluir que exista una ventanaja a favor de uno y otro sexo, al ubicarse en regiones mas o menos expuesta a la apertura comercial, ya que se observa una reduccion del premio salarial de educacion y experiencia para ambos sexos, con diferencias en algunos cuantiles de salarios.

\subsection{Descomposición del diferencial salarial en la distribución por cuantiles}

El objetivo de esta sección es realizar el análisis de descomposición del diferencial, a lo largo de toda la distribución de salarios por hora en cada uno de los cuantiles, en regiones de México, según su exposición a la apertura comercial en 2005 y 2015. 
En la gráfica 3, se muestra que en 2005 en México y las regiones que lo integran, se ha reducido la desigualdad a lo largo de la distribución de salarios, sin embargo, la mayor parte de la brecha se explica por los coeficientes y no por las dotaciones de capital humano, lo que implica presencia de discriminación.

$\mathrm{Al}$ analizar al interior de las regiones se revela que, en la zona de alta y media exposición a la apertura comercial, se observa un aumento en la parte no explicada al avanzar en los cuantiles de salario, sucede lo contrario a nivel nacional y en la región de baja exposición, ya que se revela una reducción en la parte de los coeficientes, lo que implica que hay una aumento en el valor de las características a favor de las mujeres.

Los resultados muestran la presencia "techo de cristal", en regiones de alta y media exposición a la apertura comercial, dado que, aunque, se ha reducido la desigualdad, no ha sucedido lo mismo en la parte no explicada por las características productivas, principalmente en los cuantiles altos de la distribución salarial.

\section{Gráfica 3. Descomposición del diferencial salarial por hora en la distribución por cuantiles en México y regiones según su exposición a la apertura comercial. 2005}

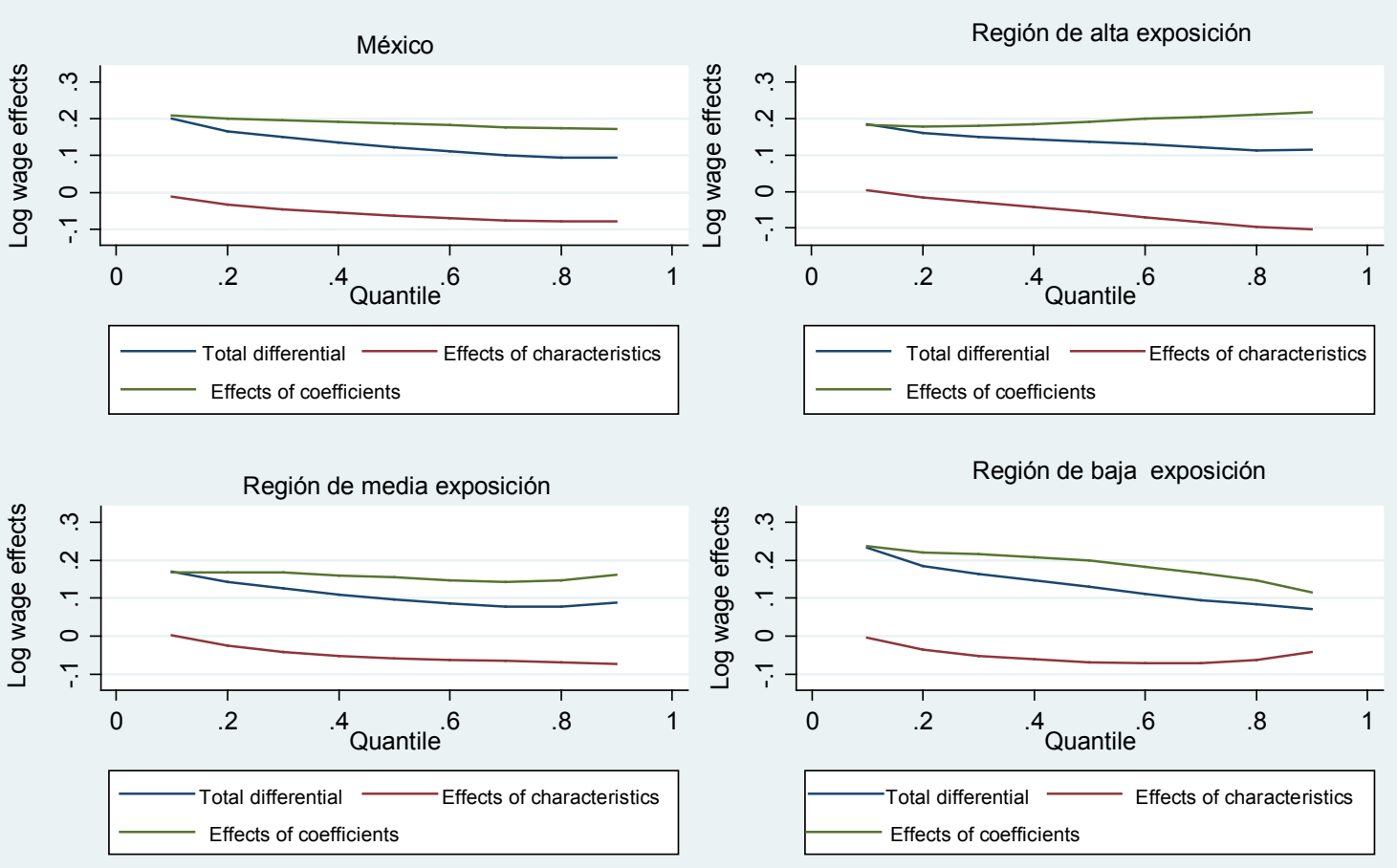

Elaboración propia en base a los datos de la ENOE 2005

En la gráfica 4, se analiza la descomposición del diferencial de salarios por hora para el 2015. De manera general se observa que para México y regiones la desigualdad cae a lo largo de la distribución salarial, asimismo, la mayor parte de la brecha se explica por el efecto de los coeficientes y no por las características de capital humano. 
Empero, para México y las regiones que lo integran se revela una reducción de la parte no explicada por las características productivas, principalmente en la región de baja exposición, donde parece haber un aumento del valor de las variables de capital humano para las mujeres, contrario a lo que sucede en la región de media exposición.

Por lo anterior se deduce, que a medida que se avanza en los cuantiles de ingreso, se reduce la desigualdad salarial, aunque la mayor parte de esta se explica por el efecto de los coeficientes y no por las características de capital humano. En la región de media exposición sigue permaneciendo el efecto "techo de cristal", no obstante, parece haber mejores condiciones salariales para las mujeres en la región de baja exposición.

\section{Gráfica 4. Descomposición del diferencial salarial por hora en la distribución por cuantiles en México y regiones según su exposición a la apertura comercial. 2015}
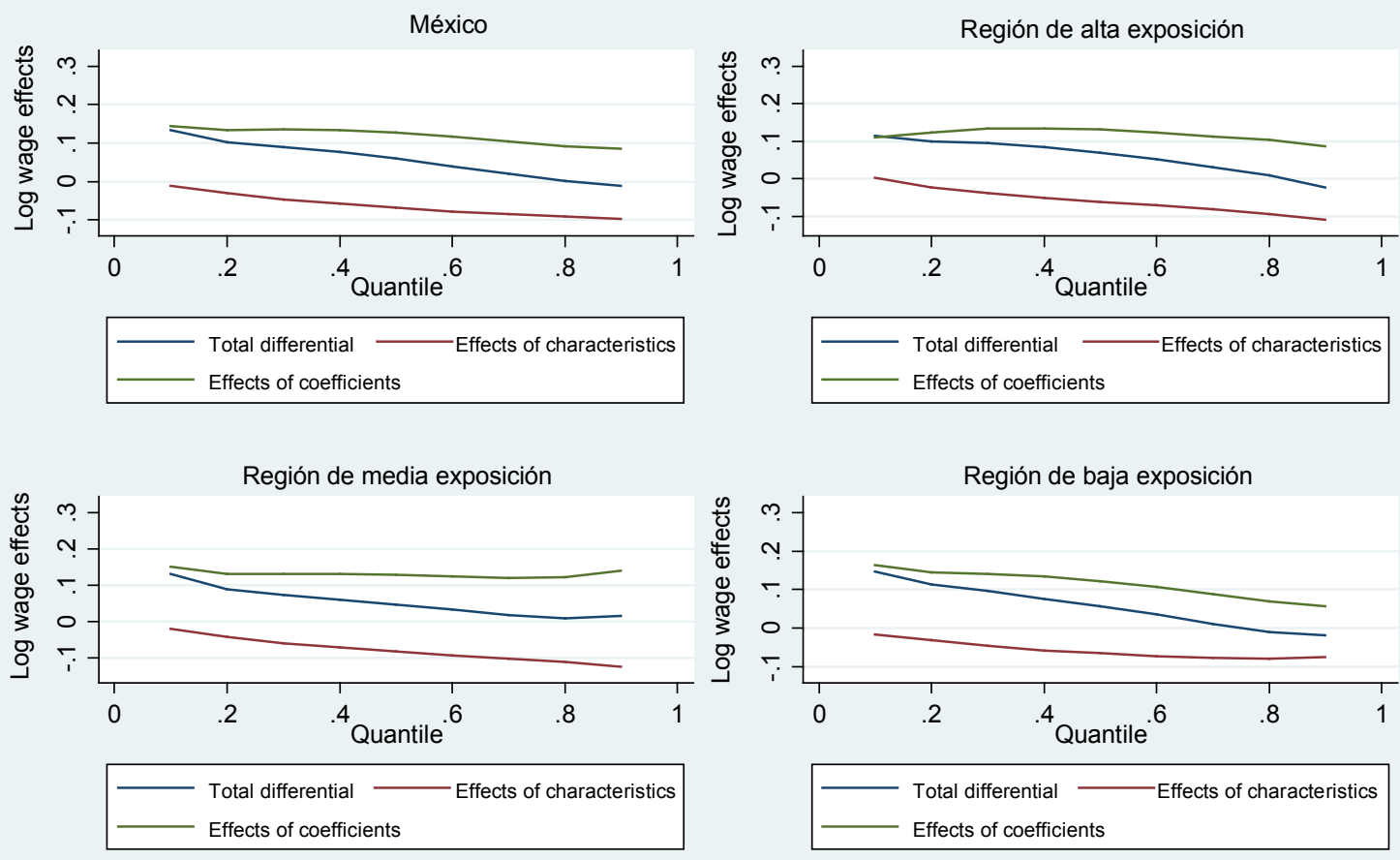

Elaboración propia en base a los datos de la ENOE 2015

De manera general se puede deducir que, en México y regiones, las brechas de salarios se han reducido de 2005 al 2015, aunque la mayor parte de las diferencias no se explican por las características productivas. Al indagar en cada uno de los años de estudio se encontró que, en 2005, las regiones de alta y media exposición se reflejan el fenómeno "techo de cristal" y esta misma situación se mantiene para la región de media exposición en 2015. Por otro lado, se revela que las mujeres parecen tener mejores condiciones salariales en la región de baja exposición a la apertura comercial, ya que es en esta zona donde se muestra un incremento del valor de las características de capital humano, por lo que estos resultados coinciden por lo encontrado por Domínguez y Brown (2010), donde revelan un impacto negativo de la orientación exportadora que tiene el país sobre los salarios de ambos géneros, sin embargo, las mujeres, muestran una mayor penalización salarial en términos absolutos como relativos. 
Asimismo, la evidencia encontrada va en línea con los hallazgos de Popli (2013), Arceo y Campos (2014), así como Tello et al. (2012), ya que indican la existencia de una disminución de la desigualdad a favor de las mujeres, aunque al descomponer la brecha en características y precios, encuentran que la mayor parte de las diferencias se debe al efecto de los precios, sin embargo, Popli revela evidencia de "techo de cristal" y Arceo y Campos, de un patrón estable de "piso pegajoso" y otro decreciente de "techo de cristal", Tello, por su parte, observa "piso pegajoso" y "techo de cristal" en sus resultados.

\section{Conclusión}

México no ha estado exento de la influencia de los mercados internacionales, abriéndose a ellos después del agotamiento del modelo económico de sustitución de importaciones a finales de los años 80s y lo cual ha tenido como resultado una nueva estructura que ha provocado importantes cambios en la economía nacional, modificando las actividades productivas, la localización de éstas, el mercado de bienes y servicios, de dinero y de trabajo.

El mercado laboral se ve afectado de manera directa, pues la reconfiguración de las regiones de México, concentrando actividades de más valor agregado en unas que en otras, lleva consigo que no todos los trabajadores sean remunerados, de la misma manera, existiendo diferencias salariales entre los trabajadores por género, además de existir diferencias entre las regiones.

Dada la importancia del tema, este trabajo se ocupa del análisis de la estructura ocupacional y la desigualdad por género en México y sus regiones según su apertura comercial entre 2005 y 2015. Los resultados revelan que se observa una mejora en los rendimientos a la educación y experiencia de las mujeres con salarios altos, aunque su nivel de participación se ha mantenido relativamente sin cambios, en el periodo analizado. Al indagar las diferencias salariales por género, se encontró que en México y sus regiones, la brecha se ha reducido, pero sigue estando en contra de las mujeres y la mayor parte de las diferencias no se explican por las características productivas.

Este estudio proporciona información interesante. Debido a que se ha encontrado gran heterogeneidad a nivel regional, resultó importante dividir el país en zonas geográficas y ver como se ha comportado la brecha en la década más reciente. Los resultados revelaron que, en 2005, las regiones de alta y media exposición se reflejan el fenómeno de "techo de cristal" y se mantiene para la última región en 2015. Asimismo, se encontró que en 2005 y 2015 la región de baja exposición a la apertura comercial, muestra reducción de la desigualdad al avanzar en los cuantiles de salario, debido a una reducción del valor de los coeficientes, lo que implica que hay un aumento en el valor de las características a favor de las mujeres. Con esto se puede deducir, que el mercado más favorable para las mujeres se encuentra en las regiones de baja exposición a la apertura comercial.

En términos generales se puede concluir que la desigualdad salarial por género ha sido un tema de preocupación constante en México y en el mundo, en el pasado y en el presente, aunque en el país la ley federal del trabajo, establece el principio de igualdad y no discriminación en contra de las mujeres y otros grupos sociales, sigue existiendo evidencia de discriminación, principalmente en las regiones más expuestas a la apertura comercial.

La contribución de esta investigación es obtener las diferencias salariales a lo largo de toda la distribución, por cuantiles, ya que la teoría económica estándar se centra en la dinámica salarial promedio 
en lugar de los cambios en toda la distribución salarial, ignorando así las diferencias en la parte inferior o la parte superior de la distribución salarial, aunado a que las estadísticas salariales promedio pueden ocultar funciones importantes en la estructura salarial, como el problema de los ingresos salariales omitidos, se detectó la necesidad, de incluir el análisis con las técnicas de imputación de ingresos laborales, dado que se ha evidenciado por parte de Campos-Vázquez (2013) y Rodríguez-Oreggia y Videla (2014) un incremento de los individuos que no dan una respuesta sobre sus ingresos laborales en la ENOE en los últimos años, principalmente en los estratos altos de salarios y educación, donde puede haber un número considerable de mujeres que no estamos incluyendo en la muestra.

\section{Referencias}

Aguilera, Albany y David Castro Lugo. 2016. La dinámica regional y el proceso de apertura comercial en México. En Mercado laboral en México: situación y desafíos, compilado por David Castro y Reyna Rodríguez. Universidad Autónoma de Coahuila: De Laurel, 49-80.

Arceo Eva y Raymundo Campos. 2014. Evolución de la brecha salarial de género en México. El Trimestre Económico, 81(3): 619-653.

Artecona, Raquel y Wendy Cunningham. 2002. Effects of trade liberalization on the gender wage gap in Mexico. The World Bank. Development Research Group/ Poverty Reduction and Economic Management Network, 1-28.

Becker, Gary. 1987. Tratado sobre la familia. Madrid: Alianza Universidad.

Blau, Francine y Lawrence Kahn. 1996. Wage structure and gender earnings differentials: An internationals comparison. Economics, 63(250): 29-62. DOI: https://doi.org/10.2307/2554808

Blinder, Alan. 1973. Wage discrimination: reduced forms and structural estimates. The Journal of Human Resources, 8(4): 436-455. DOI: https://doi.org/10.2307/144855

Campos-Vázquez, Raymundo. 2013. Efectos de los ingresos no reportados en el nivel y tendencia de la pobreza laboral en México. Ensayos Revista de Economía, 32(2): 23-54.

Castro David y Luis Huesca. 2007. Desigualdad salarial en México: Una revisión. Papeles de población, 14 (54): 225-264. DOI: http://dx.doi.org/10.20983/noesis.2015.1.2

Del Río, Carlos y Olga Cantó. 2008. Pobreza y discriminación salarial por razón de género en España. Revista de Economía Pública, 184 (1): 67-98.

DiNardo, Jhon, Nicole Fortin y Thomas Lemieux. 1996. Labor market institutions and the distribution of wages, 1973-1992. A semiparametric approach. Econométrica, 64(5): 1001-1044. https://doi. org/10.2307/2171954

Domínguez, Lilia y Flor Brown. 2010. Trade Liberalization and gender wage inequality in Mexico, Feminist Economics, 16 (4): 53-79. https://doi.org/10.1080/13545701.2010.530582

Dueñas, Diego, Carlos Iglesias y Raquel Llorente. 2015. Abordando la desigualdad de género. Empleo en técnicas de información y la comunicación y diferencias salariales por género en España. Ensayos Sobre Política Económica, 33(78): 207-2019.

Enamorado, Ted, Ana Izaguirre y Hugo Ñopo. 2009. Gender wage gaps in central american countries: Evidence from a non-parametric approach. Inter-American Development Bank. Working Papers Series 111: 1-32. 
Galvis, Luis. 2011. Diferencias salariales por género y región en Colombia: Una aproximación con regresión por cuentiles. Revista de Economía del Rosario, 13(2): 235-277.

Gasparini, Leonardo. 2006. La distribución del ingreso: un breve resumen en tres décadas. Económica, 52(1-2): 53-76.

Gutiérrez, Luis. 2008. La distribución del ingreso en México: un análisis regional. 1990-2004. Problemas del Desarrollo. Revista Latinoamericana de Economía, 39 (152). 139-163.

Hanson, Gordon. 2005. Globalization, labor income, and poverty in Mexico. NBER Working Paper Series, 1-50. https://doi.org/10.3386/w11027

Heckman, James. 1979. Sample selection bias as a specification error. Econometrica. 47(1):153-161. https://doi.org/10.2307/1912352

Hirsch, Boris. 2013. The impact of female managers on the gender pay gap: Evidence from linked employer-employee data for germany. Economics Letters, 119(3): 348-350.

https://doi.org/10.1016/j.econlet.2013.03.021

Instituto Nacional de Estadística y Geografía (2010). Censo de Población. INEGI, Aguascalientes, México.

Instituto Nacional de Estadística y Geografía. 2005-2015. Encuesta Nacional de Ocupación y Empleo. INEGI, Aguascalientes, México.

Juhn, Chinhui, Kevin Murphy y Pierce Brooks. 1991. Accounting for the slowdown in black-white convergence. En Workers and their wages, editado por M. Osters. American Enterprise Institute Press.

Juhn, Chinhui, Kevin Murphy y Pierce Brooks. 1993.Wage inequality and the rise in returns to skill. Journal of Political Economy, 101 (31): 410-442. https://doi.org/10.1086/261881

Koenker, Roger y Gilbert Basset. 1978. Regression quantiles. Econometrica, 46(1): 33-50. https://doi. org/10.2307/1913643

Koenker, Roger y Kevin Hallock. 2001. Quantile regression. Journal of Economic Perspectives, 15(4):143156. https://doi.org/10.1257/jep.15.4.143

Liu, Pak-Wai, Xin Meng y Junsen Zhang. 2000. Sectoral gender wage differentials and discrimination in the transitional chinese economy. Journal of Population Economics, 13(2): 331-52. https://doi.org/10.1007/ s001480050141

Machado, Jose y Jose Mata. 2005. Counterfactual decomposition of changes in wage distributions using quantile regression. Journal of Applied Econometrics, 20 (4): 445-65. https://doi.org/10.1002/jae.788

Martínez-Jasso, Irma y Gloria Acevedo-Flores. 2004. La brecha salarial en México con enfoque de género: Capital humano, discriminación y selección muestral. Ciencia, 7(1): 66-71.

McCulloch, Rachel. 2006. Protection and real wages: The Stolper-Samuelson theory. En Samuelsonian economics and the twenty-first century, editado por Michael Szenberg, Lall Ramrattan, y Aron Gottesman. NuevaYork: Oxford University Press.

Melly Blaise. 2005. Descomposition of diferences indistribution using quiantile regression. Labour Economic, 12(4): 577-590. https://doi.org/10.1016/j.labeco.2005.05.006

Mendoza, Eduardo y Karina García. 2009. Discriminación salarial por género en México. Problemas del Desarrollo, 40 (156): 77-99.

Mincer, Jacob. 1974. Schooling, experience, and earnings. National Bureau of Economic Research. Nueva York: Columbia University Press. 
Mysikova, Martina. 2012. Gender wage gap in the Czech Republic and central european countries. Prague Economic papers, 21(3): 328-346. https://doi.org/10.18267/j.pep.427

Neary, Peter (2004). The Stolper-Samuelson theorem. En Encyclopedia of world trade since 1450, editado por John McCusker. Nueva York: Macmillan Reference, 719-720.

Nopo, Hugo, Nancy Daza y Johanna Ramos. 2011. Gender earnings gaps in the world. Institute for the study of labor (IZA).

Oaxaca, Ronald. 1973. Male-female wage differentials in urban labour markets. International Economic Review, 14 (3): 693-709.

Popli, Gurleen. 2013. Gender wage differentials in México: A distributional approach. Journal of the Royal Statistical Society, 176 (2): 295-319.

Rodríguez, Reyna y David, Castro. 2014a. Discriminación salarial de la mujer en el mercado laboral de México y sus regiones. Economía Sociedad y Territorio. 14 (46): 655-686. https://doi.org/10.22136/ est002014392

Rodríguez, Reyna y David, Castro. 2014b. Análisis de la discriminación salarial por género en Saltillo y Hermosillo: un estudio comparativo en la industria manufacturera. Nóesis. Revista de Ciencias Sociales y Humanidades, 23(46): 80-113.

Rodríguez-Oreggia Eduardo y Bruno Videla. 2014. Imputación de ingresos laborales: una aplicación con encuestas de empleo en México. Banco de México. Documentos de investigación, 21:1-37.

Semykina, Anastasia y Susan Linz. 2010. Analyzing the gender pay gap in transition economies: how much does personality matter?. Human Relations, 63(4) : 447-469. https://doi.org/10.1177/0018726709339094 Tello, Claudia, Raúl Ramos y Manuel Artís. 2012. Changes in wage structure in México going beyond the mean: An analysis of differences in distribution, 1987-2008. Working paper, 24:1-43. https://doi. org/10.2139/ssrn.2060555

Wolsczak-Derlacz, Joana. 2013. Mind the gender wage gap the impact of trade and competition on sectoral wage differences. The World Economy 36(4): 437-464. https://doi.org/10.1111/twec.12026 